\title{
Temperature-induced transition of magnetic anisotropy between in-plane and out-of-plane directions in GaMnAs film
}

\author{
Sangyeop Lee ${ }^{\mathrm{a}}$, Seonghoon $\mathrm{Choi}^{\mathrm{a}}$, Seul-Ki Bac ${ }^{\mathrm{a}}$, Hakjoon Lee ${ }^{\mathrm{a} \#}$, Taehee Yoo ${ }^{\mathrm{a}}$, \\ Sanghoon Lee $\mathrm{a}^{\mathrm{a}^{*}}$, \\ X. Liu ${ }^{\mathrm{b}}$, and J. K. Furdyna ${ }^{\mathrm{b}}$ \\ ${ }^{a}$ Physics Department, Korea University, Seoul 136-713, Korea \\ ${ }^{\mathrm{b}}$ Physics Department, University of Notre Dame, Notre Dame, Indiana 46556, USA
}

\begin{abstract}
We used the Hall effect and magnetization measurements to investigate the temperature dependence of the magnetic anisotropy of a ferromagnetic semiconductor GaMnAs film grown on a (001) GaAs substrate. The Hall effect was systematically measured by applying an external magnetic field within and normal to the film plane. The switching behavior of the magnetization during the reversal process revealed the coexistence of in-plane and out-of-plane magnetic anisotropies in the film. However, these two types of magnetic anisotropies strongly depended on the temperature. Specifically, the out-of-plane anisotropy was dominant in the low-temperature region (i.e., 3-10 K), whereas the in-plane anisotropy became dominant in the temperature region higher than $15 \mathrm{~K}$. This temperature dependent change in the magnetic anisotropy was further confirmed using direct magnetization measurements.
\end{abstract}

Ferromagnetic semiconductor, Magnetic anisotropy, Planar Hall Effect

*E-mail: slee3@korea.ac.kr

\# E-mail: grayun@empal.com 


\section{Introduction}

The magnetic anisotropic properties of GaMnAs ferromagnetic films have received much attention because of their potential applications in practical spin memory devices[1]. It is known from earlier studies that the magnetic anisotropy of a GaMnAs film depends on many material parameters, including the strain, alloy composition, and carrier concentration [2-6]. In particular, the magnetic anisotropy (either in-plane or out-of-plane) of a GaMnAs film strongly depends on the strain within the film [7-9]. For example, although the in-plane magnetic anisotropy is dominant in a GaMnAs film under a compressive strain, the out-of-plane anisotropy is dominant when it is under a tensile strain.

Because of the easy growth of a GaMnAs film on a GaAs substrate, in which the film is under a compressive strain, the in-plane magnetic anisotropy has primarily been investigated. Numerous experimental studies have revealed the complex nature of the magnetic anisotropies of a GaMnAs film within the film plane. For example, cubic anisotropy in the $\langle 100\rangle$ directions, uniaxial anisotropy in the <110> directions, and another uniaxial anisotropy in the [100] direction have been detected in a GaMnAs film [10-16]. These anisotropies show dependence not only on the material parameters, as previously mentioned, but also on the temperature [17, 18]. Many investigations have shown that the dominant magnetic anisotropy changes from cubic to uniaxial with increasing temperature [10, 19].

Although the investigations of the magnetic anisotropy within the (100) plane have been intense, not much attention has been given to the out-of-plane magnetic anisotropy of a GaMnAs film. Furthermore, the temperature-induced transition of the magnetic anisotropy between the in-plane and out-of-plane directions has never been reported for a GaMnAs film. In this study, we investigated the temperature dependent change in the magnetic anisotropy between the in-plane and out-of-plane directions in a GaMnAs film. For this purpose, we adopted direct magnetization measurements and Hall effect measurements, which are very sensitive to the direction of the magnetization in a magnetic film and thus provided a straightforward method for obtaining information about the magnetic anisotropy of the GaMnAs film.

\section{Experiments}

A ferromagnetic GaMnAs film was grown on a (001) GaAs substrate using a low substrate temperature of $250^{\circ} \mathrm{C}$ in a RIBER R\&D 32 molecular beam epitaxy system equipped with Ga, As, and Mn as elemental sources. A 10-nm GaAs layer was first grown on the substrate at a low temperature, followed by the deposition of a $75-\mathrm{nm} \mathrm{Ga}_{1-\mathrm{x}} \mathrm{Mn}_{\mathrm{x}} \mathrm{As}$ film (with $\mathrm{x}=0.02$ ). The 
growth of the GaMnAs film was monitored using reflection high-energy electron diffraction (RHEED). The RHEED pattern was streaky throughout the film deposition, indicating smooth two-dimensional growth. A $5 \times 5 \mathrm{~mm}^{2}$ square piece was then cleaved from the wafer for the fabrication of the Hall device. A $300 \times 1500 \mu \mathrm{m}^{2}$ rectangular Hall device with a long dimension in the $[1 \overline{1} 0]$ direction was finally patterned on the cleaved piece using photolithography and chemical wet etching.

Transport measurements were performed using a direct current of $20 \mu \mathrm{A}$ along the [1 10$]$ direction, which was used as a sensing current for detecting the Hall resistance. The Curie temperature $T_{\mathrm{C}}$ of the film was estimated from the magnetization measurements, which showed an increase in magnetization near $25 \mathrm{~K}$. The angular dependence of the Hall effect was measured by mounting the sample on a special holder designed to allow the applied magnetic field to be rotated within and out of the film plane. In discussing the configuration of the experiment, we use the $\theta_{H}$ and $\varphi_{H}$ angles to define the direction of the external field, and $\theta_{M}$ and $\varphi_{M}$ to indicate the direction of the magnetization in the film, as shown in Fig. 1. The $\theta_{H}$ and $\theta_{M}$ angles were measured from the [001] direction (i.e., the normal to the film plane); $\varphi_{H}$ and $\varphi_{M}$ were measured counterclockwise from the [1 $\left.\overline{1} 0\right]$ direction in the (001) plane.

\section{Results and discussion}

The Hall effect measurement is known to be especially useful for studying the magnetic anisotropic properties of ferromagnetic materials because of its sensitive dependence on the direction of the magnetization, as given by the following[20]:

$$
R_{H}=\frac{R_{0}}{t} H \cos \theta_{H}+\frac{R_{S}}{t} M \cos \theta_{M}+\frac{k}{t} M^{2} \sin ^{2} \theta_{M} \sin 2 \varphi_{M} \text { (1), }
$$

where the first, second, and third terms represent the normal, anomalous, and planar Hall resistances, respectively. In the case of a ferromagnetic material, the normal Hall resistance (NHR) is negligible compared to the anomalous Hall resistance (AHR) and planar Hall resistance (PHR). Therefore, in this work, only the AHR and PHR will be considered in discussing the Hall effect data measured on the ferromagnetic GaMnAs film. In Eq. (1), $R_{0}$ and $R_{\mathrm{S}}$ are the normal and anomalous Hall coefficients, respectively, and $k$ is a constant related to the anisotropic magnetoresistance. Finally, $M$ and $t$ are the magnetization and thickness of the film, respectively.

As seen in Eq. (1), the value of the Hall resistance is a function of the direction of 
magnetization $\theta_{M}$ and $\varphi_{M}$. In the case of a ferromagnetic film with in-plane easy axes, the anomalous Hall effect becomes zero (i.e., $\theta_{M}=90^{\circ}$ ) when the external field is applied in the film plane, and the changes in the magnetization within the film plane are then reflected only in the planar Hall effect (i.e., in the third term of Eq. (1)). Therefore, planar Hall effect (PHE) measurements can be used to monitor the direction of the magnetization within the film plane during the magnetization reversal and to study the details of the in-plane magnetic anisotropy [21-23]. Specifically, the angular dependence of the PHR can be used to determine the directions of the magnetic easy and hard axes of the film.

We first started the Hall measurements at $3 \mathrm{~K}$ to identify the magnetic anisotropic properties of the GaMnAs film. The PHR (i.e., with an in-plane applied field) was measured by rotating the direction of the field while keeping its strength constant. The observed dependences of the PHR on the field angle at field strengths between 250 and 3000 Oe are plotted in Fig. 2. The PHR data obtained with a magnetic field larger than 1000 Oe vary periodically between $+75 \Omega$ and $-75 \Omega$, with a rather abrupt transition near $\varphi_{H}=0, \varphi_{H}=90^{\circ}$, and $\varphi_{H}=180^{\circ}$. This is a typical angle-dependent behavior of the PHR for a GaMnAs film with in-plane magnetic anisotropy, indicating the presence of two nearly orthogonal magnetic easy axes along the $\langle 100\rangle$ directions and two hard axes along the $\langle 110\rangle$ directions in the film plane.

However, the amplitude of the PHR decreases dramatically as the field strength is reduced to 500 Oe and below. This is due to the presence of areas with strong out-of-plane anisotropy and pinning field distributions even within the in-plane magnetic domains $[18,21$, 24]. When a strong external field is applied along the film plane, total magnetization of the sample will align with the field and follow the direction of the field when it rotates. However, when a small field strength is used, the magnetization in the areas with a vertical easy axis will remain in the vertical direction rather than follow the rotation of the field. Furthermore, when the external field is rotated in the film plane, the magnetic energy barrier between the two easy axes must be overcome by the external field even for the transition of the magnetization within the film plane. It is now well known that the magnetic pinning energy, which corresponds to the magnetic energy barrier between the easy axes, is different for each domain in a GaMnAs film and follows a Gaussian-like distribution[24]. The fraction of magnetic domains participating in the reorientation process will then depend on the external field strength. Specifically, when the field strength is strong enough to cover the entire range of the pinning field distribution, the entire in-plane area follows the magnetization reversal process when the field is rotated. However, if the field is reduced below the high end of the pinning field distribution of the 
sample, the fraction of domains participating in the rotation begins to decrease. This will continue until the applied field strength reaches the lower boundary of the pinning field distribution, at which no domain will undergo the magnetization reorientation when the field direction is rotated. Such a relaxation of the vertical magnetization and reduction of the in-plane domains participating in the reorientation process as the field strength is reduced is reflected in the conspicuous reduction in the amplitude of the planar Hall resistance during the reversal process (see the top two panels of Fig. 2).

In order to investigate the magnetic anisotropy along the out-of-plane direction, we rotated the field direction in the $(1 \overline{1} 0)$ plane at a constant field strength. The angular dependences of the Hall resistance obtained with field strengths between 500 Oe and 2000 Oe are shown in Fig. 3. In the case of a small magnetic field of $500 \mathrm{Oe}$, an abrupt transition occurs after passing the film plane for both the clockwise $(\mathrm{CW})$ and counterclockwise $(\mathrm{CCW})$ field rotations. As the field increases to $1000 \mathrm{Oe}$, the value of PHR begins to change even before the field direction crosses the film plane (i.e., before $\theta_{H}=90^{\circ}$ ). This tendency is further enhanced when the field strength increases to $2000 \mathrm{Oe}$, as seen in the bottom panel of Fig. 3. Furthermore, the hysteresis width between the CW and CCW rotations systematically decreases with increasing field strength. This phenomenon is due to the Zeeman effect, which increases with the field strength. The Hall measurements obtained with field rotation out of the film plane clearly indicate that the GaMnAs film under investigation has areas with the out-of-plane magnetic easy axis. The results of the Hall resistance measurements performed while rotating the field within and out-of the film plane indicate the coexistence of magnetic domains with inplane and out-of-plane magnetic anisotropies in our GaMnAs film at a temperature of $3 \mathrm{~K}$.

We further investigated the magnetic anisotropy of the film by performing a field scan of the Hall resistance at a fixed external field direction. The Hall resistance data obtained with the applied field within the film plane (i.e., $\theta_{H}=90^{\circ}$ ) and nearly normal to the film plane (i.e., $\theta_{H}=0^{\circ}$ ) at several different temperatures are shown in the $1^{\text {st }}$ and $2^{\text {nd }}$ rows of Fig. 4 , respectively. In the case of the in-plane measurements (i.e., the planar Hall resistance measurements), $\varphi_{H}=10^{\circ}$ and $\varphi_{H}=170^{\circ}$ were selected as two field directions, which were symmetric to each other with respect to the [110] in-plane magnetic hard axis, to identify the in-plane magnetization behavior. The PHR data show a two-step transition behavior during the reversal process for both field directions at all temperatures. However, in the PHR data taken at $3 \mathrm{~K}$, an abrupt transition occurs with a negative coercive field(i.e., the transition occurs even before the field direction reversal), as indicated by the dotted lines in the first two panels of the 
$1^{\text {st }}$ row of Fig. 4.This abnormal behavior observed in the PHR of the GaMnAs film was due to the transition of the vertical component of the magnetization[25, 26]. In the PHR experiment, when the magnetic field was strong in the film plane, it directed the magnetization of the sample in the in-plane direction, including areas having out-of-plane easy axes. However, as the field strength was reduced, areas with out-of-plane anisotropy returned to their own easy magnetic axis, which was out-of-plane, even before the field direction was reversed. This appears as a negative cohesive field in the PHR data taken at 3 and $10 \mathrm{~K}$ (see the first two panels of the $1^{\text {st }}$ row of Fig. 4). Interestingly, such a vertical transition of the magnetization is significant and overshadows the signal from the in-plane transition of the magnetization (i.e., the normal PHR). This indicates that the magnetization along the normal to the film plane was much more preferred at 3 and $10 \mathrm{~K}$ in the sample, which implies the dominance of the out-of-plane magnetic anisotropy at low temperature. However, such a negative coercive field decreased as the temperature increased, and the PHR data became nearly normal for the film with the inplane magnetization, as shown in the third panel of the first row in Fig.4. This is a clear indication of the magnetic anisotropy change from out-of-plane dominant to in-plane dominant as the temperature increases.

The magnetic anisotropy change with the temperature is also seen in the AHR data plotted in the $2^{\text {nd }}$ row of Fig. 4 . The data taken at 3 and $10 \mathrm{~K}$ show an abrupt transition with a single hysteresis centered at the zero field. This is a typical AHR behavior of a ferromagnetic film with an out-of-plane magnetic easy axis, and thus supports the presence of magnetic domains with a strong out-of-plane anisotropy in the film. This result is consistent with the fact that a negative coercive field appeared in the in-plane measurements shown in the first and second panels of the $1^{\text {st }}$ row of Fig. 4 . The AHR data also undergo a significant change as the temperature increases. This is clearly seen in the $2^{\text {nd }}$ row of Fig. 4 , where the square hysteresis of the AHR with an abrupt transition at $3 \mathrm{~K}$ and $10 \mathrm{~K}$ changes to a monotonic behavior with a gradual variation of the Hall resistance values at $15 \mathrm{~K}$ and $20 \mathrm{~K}$. Such a gradual variation of the AHR during the field sweep is a typical behavior that can be observed in a ferromagnetic film with in-plane magnetic easy axes. The PHR and AHR results both consistently indicate that the magnetic anisotropy of our GaMnAs film changed from out-of-plane dominant to in-plane dominant as the temperature increased. Specially, the significant difference between the data taken below and above $15 \mathrm{~K}$ for both the PHR and AHR is a key signature to identifying the anisotropy change with an increasing temperature. This temperature dependent anisotropy transition from the out-of-plane dominant to the in-plane dominant with an increasing temperature is consistent with the magnetization data and will be discussed below. 
The transition of the magnetic anisotropy with increasing temperature must be reflected in the in-plane and out-of-plane components of the magnetization, which can be directly measured using a vibrating sample magnetometer (VSM). The temperature dependent magnetization data are plotted in Fig. 5, where the solid circles and open squares represent the magnetizations along the $[100]$ and $[1 \overline{1} 0]$ directions, respectively, (i.e., the in-plane magnetization of the film), and the solid squares represent the magnetization along the [001] direction (i.e., the out-of-plane magnetization of the film). All of the data show that the sample starts to be magnetized at a temperature of around $25 \mathrm{~K}$, which is the Curie temperature of this film, and the magnetization monotonically increases at the same speed in all directions as the temperature decreases close to $17 \mathrm{~K}$. However, the in-plane magnetizations for both the [100] and [1 $\overline{10}$ ] directions decrease as the temperature decreases below $17 \mathrm{~K}$. In contrast, the magnetization along the [001] direction continuously increases even below $17 \mathrm{~K}$. These opposite behaviors between the in-plane and out-of-plane magnetizations below $17 \mathrm{~K}$ are the signature of a temperature-induced magnetic anisotropy transition between in-plane and out-of-plane anisotropies. The temperature behaviors of the magnetizations are consistent with the temperature-dependent change in the magnetic anisotropy observed in the previously discussed Hall resistance measurements. Therefore, both the Hall effect and magnetization measurements consistently revealed the magnetic anisotropy change in our GaMnAs film from out-of-plane dominant to in-plane dominant as the temperature increased.

Such conversion of the dominant magnetic anisotropy in the GaMnAs film from the out-ofplane to the in-plane direction may result from the relative strength of the anisotropies depending on the temperature. The magnetic anisotropy of the GaMnAs film is known to be quite complex, consisting of cubic and uniaxial anisotropies that depend on various parameters, such as strain, Mn concentration, carrier concentration[2-4, 27]. Therefore, it is very difficult to point out a specific reason because some of those parameters in the GaMnAs film vary together as temperature changes. However, an investigation of the temperature dependence of the magnetic anisotropy revealed that the in-plane uniaxial anisotropy is rather insensitive to the temperature while the cubic anisotropy changes quite rapidly with an increasing temperature. $[10,19]$ Such temperature stability of the in-plane uniaxial anisotropy may be one of the reasons for the in-plane dominant behavior of our GaMnAs film at high temperatures. The PHR behavior observed at $20 \mathrm{~K}$ (see the last panels in the $1^{\text {st }}$ row of Fig. 4) indeed shows the feature arising from the GaMnAs film with a dominant uniaxial in-plane anisotropy $[19,24]$.

\section{Conclusions}


We investigated the magnetic anisotropy of a GaMnAs film and its temperature dependence using Hall effect and magnetization measurements. The experiment showed features that originated from the coexistence of magnetic domains with out-of-plane and inplane anisotropies in the film. The temperature dependence of the Hall effect measurements revealed that the magnetic anisotropy of the film changed from a state where the out-of-plane anisotropy was dominant at low temperatures to one where the in-plane anisotropy was dominant at high temperatures. Such a change in the magnetic anisotropy with temperature was further confirmed by the direct magnetization measurements. This study showed that our GaMnAs film consisted of two types of magnetic domains: one characterized by a magnetic anisotropy along the out-of-plane direction and the other by an in-plane anisotropy. The dominant magnetic anisotropy of the film switched from the out-of-plane to in-plane direction as the temperature increased.

\section{Acknowledgments}

This research was supported by the Converging Research Center Program through the Ministry of Science, ICT and Future Planning (NRF-2014M3C1A8053744); by Basic Science Research Program through the National Research Foundation of Korea(NRF) funded by the Ministry of Education (2015R1D1A1A01056614); by Korea University through a grant; and by the National Science Foundation (grant no. DMR14-00432).

\section{FIGURE CAPTION}

Fig. 1. Schematic diagram of Hall device patterned on GaMnAs film. The directions of the external field and magnetization are shown with arrows. The measurement schemes for the angles defining the field and magnetization directions are also shown, together with crystallographic directions.

Fig. 2. Angular dependence of planar Hall resistance (PHR) obtained for several different magnetic fields. The open(red) and solid(black) circles show data obtained by CW and CCW rotations of the field, respectively. The PHR shows abrupt transitions between the two PHR 
values near the $\langle 110\rangle$ direction, indicating the presence of in-plane magnetic anisotropy with four-fold symmetry in the GaMnAs film.

Fig. 3. Angular dependence of Hall resistance for $\mathrm{Ga}_{0.98} \mathrm{Mn}_{0.02} \mathrm{As}$ film obtained by changing $\theta_{H}$ at three different fixed magnetic field strengths. An abrupt one-step switching behavior in the data with $\mathrm{H}=500$ Oe changes to a less abrupt transition with a field lager than $\mathrm{H}=1000$ Oe. This is a result of the increased influence of the Zeeman effect at a larger field.

Fig. 4. Field scans of PHR ( $1^{\text {st }}$ row) and AHR ( $2^{\text {nd }}$ row) obtained at several different temperatures. The PHR values were obtained for two field directions $\left(\varphi_{H}=10^{\circ}\right.$ and $\left.\varphi_{H}=170^{\circ}\right)$, and the directions of the field sweep are indicated by arrows. The PHR data show a two-step switching behavior, indicating the presence of two in-plane magnetic easy axes. The transitions at negative coercive fields in the reversal process shown in the two left panels in the $1^{\text {st }}$ row correspond to the transitions of the out-of-plane magnetization. The AHR values in the $2^{\text {nd }}$ row show a typical switching behavior of the magnetic film with the out-of-plane easy axes at low temperatures $(3 \mathrm{~K}$ and $10 \mathrm{~K}$ ), but change to the behavior of the in-plane magnetic easy axes at $15 \mathrm{~K}$ and $20 \mathrm{~K}$.

Fig. 5. Magnetizations measured by VSM for GaMnAs film in three different field directions. When the field is applied in the sample plane (Open circle and solid circle), the magnetization increases as the temperature decreases in the temperature region above $17 \mathrm{~K}$. However, it decreases as the temperature further decreases below $17 \mathrm{~K}$. When the field is applied in the outof-plane direction (Solid square), the magnetization increases monotonically as the temperature decreases. These magnetization behaviors indicate that the dominant magnetization of the film switches between the in-plane and out-of-plane directions at a critical temperature near $17 \mathrm{~K}$.

\section{References}

[1] G.A. Prinz, Science, 282 (1998) 1660-1663.

[2] X. Liu, Y. Sasaki, J.K. Furdyna, Phys. Rev. B, 67 (2003) 205204.

[3] T. Yamada, D. Chiba, F. Matsukura, S. Yakata, H. Ohno, Phys. Status Solidi (c), 3 (2006) 4086-4089.

[4] S.Y. Yea, S.J. Chung, H. Son, D.Y. Shin, S. Lee, X. Liu, J.K. Furdyna, Solid State Commun., 147 (2008) 309-312.

[5] S. Kim, H. Lee, T. Yoo, S. Lee, S. Lee, X. Liu, J.K. Furdyna, J. Appl. Phys., 107 (2010) 
103911.

[6] K. Hamaya, T. Watanabe, T. Taniyama, A. Oiwa, Y. Kitamoto, Y. Yamazaki, Phys. Rev. B, 74 (2006) 045201.

[7] G.P. Moore, J. Ferre, A. Mougin, M. Moreno, L. Daweritz, J. Appl. Phys., 94 (2003) 4530-4534.

[8] K.Y. Wang, M. Sawicki, K.W. Edmonds, R.P. Campion, S. Maat, C.T. Foxon, B.L. Gallagher, T. Dietl, Phys. Rev. Lett., 95 (2005) 217204.

[9] S.R. Dunsiger, J.P. Carlo, T. Goko, G. Nieuwenhuys, T. Prokscha, A. Suter, E. Morenzoni, D. Chiba, Y. Nishitani, T. Tanikawa, F. Matsukura, H. Ohno, J. Ohe, S. Maekawa, Y.J. Uemura, Nature Mater., 9 (2010) 299-303.

[10] U. Welp, V.K. Vlasko-Vlasov, X. Liu, J.K. Furdyna, T. Wojtowicz, Phys. Rev. Lett., 90 (2003) 167206.

[11] K. Hamaya, T. Taniyama, Y. Kitamoto, T. Fujii, Y. Yamazaki, Phys. Rev. Lett., 94 (2005) 147203.

[12] U. Welp, V.K. Vlasko-Vlasov, A. Menzel, H.D. You, X. Liu, J.K. Furdyna, T. Wojtowicz, Appl. Phys. Lett., 85 (2004) 260-262.

[13] J. Won, J. Shin, S. Lee, T. Yoo, H. Lee, S. Lee, X.Y. Liu, J. Furdyna, Appl. Phys. Express, 6 (2013).

[14] S.K. Bac, H. Lee, S. Lee, S. Choi, T. Yoo, S. Lee, X.Y. Liu, J. Furdyna, Appl. Phys. Express, 8 (2015).

[15] K. Pappert, C. Gould, M. Sawicki, J. Wenisch, K. Brunner, G. Schmidt, L.W. Molenkamp, New J. Phys., 9 (2007) 354.

[16] K. Pappert, S. Humpfner, J. Wenisch, K. Brunner, C. Gould, G. Schmidt, L.W. Molenkamp, Appl. Phys. Lett., 90 (2007) 062109.

[17] V.G. Storchak, D.G. Eshchenko, E. Morenzoni, T. Prokscha, A. Suter, X.Y. Liu, J.K. Furdyna, Phys. Rev. Lett., 101 (2008) 027202.

[18] J. Kim, T. Yoo, S. Chung, S. Lee, X. Liu, J.K. Furdyna, J. Appl. Phys., 105 (2009) $07 \mathrm{C501.}$

[19] D.Y. Shin, S.J. Chung, S. Lee, X. Liu, J.K. Furdyna, Phys. Rev. B, 76 (2007) 035327.

[20] H. Son, S.J. Chung, S.Y. Yea, S. Lee, X. Liu, J.K. Furdyna, J. Appl. Phys., 103 (2008) $07 F 313$.

[21] J. Kim, D.Y. Shin, T. Yoo, H. Kim, S. Lee, X. Liu, J.K. Furdyna, J. Appl. Phys., 103 (2008) 07D101.

[22] H. Lee, S. Chung, S. Lee, X. Liu, J.K. Furdyna, Solid State Commun., 149 (2009) 1300-1303.

[23] D.Y. Shin, S.J. Chung, S. Lee, X. Liu, J.K. Furdyna, IEEE Trans. Magn., 43 (2007) 3025-3027.

[24] J. Kim, D.Y. Shin, S. Lee, X. Liu, J.K. Furdyna, Phys. Rev. B, 78 (2008) 075309. 
[25] S. Lee, H. Lee, T. Yoo, S. Lee, X. Liu, J.K. Furdyna, J. Cryst. Growth, 378 (2013) 337-341.

[26] H. Son, S.J. Chung, S.Y. Yea, S. Lee, X. Liu, J.K. Furdyna, J. Appl. Phys., 103 (2008).

[27] S. Lee, H. Lee, T. Yoo, S. Lee, X. Liu, J.K. Furdyna, J. Appl. Phys., 113 (2013) $17 \mathrm{C} 706$. 


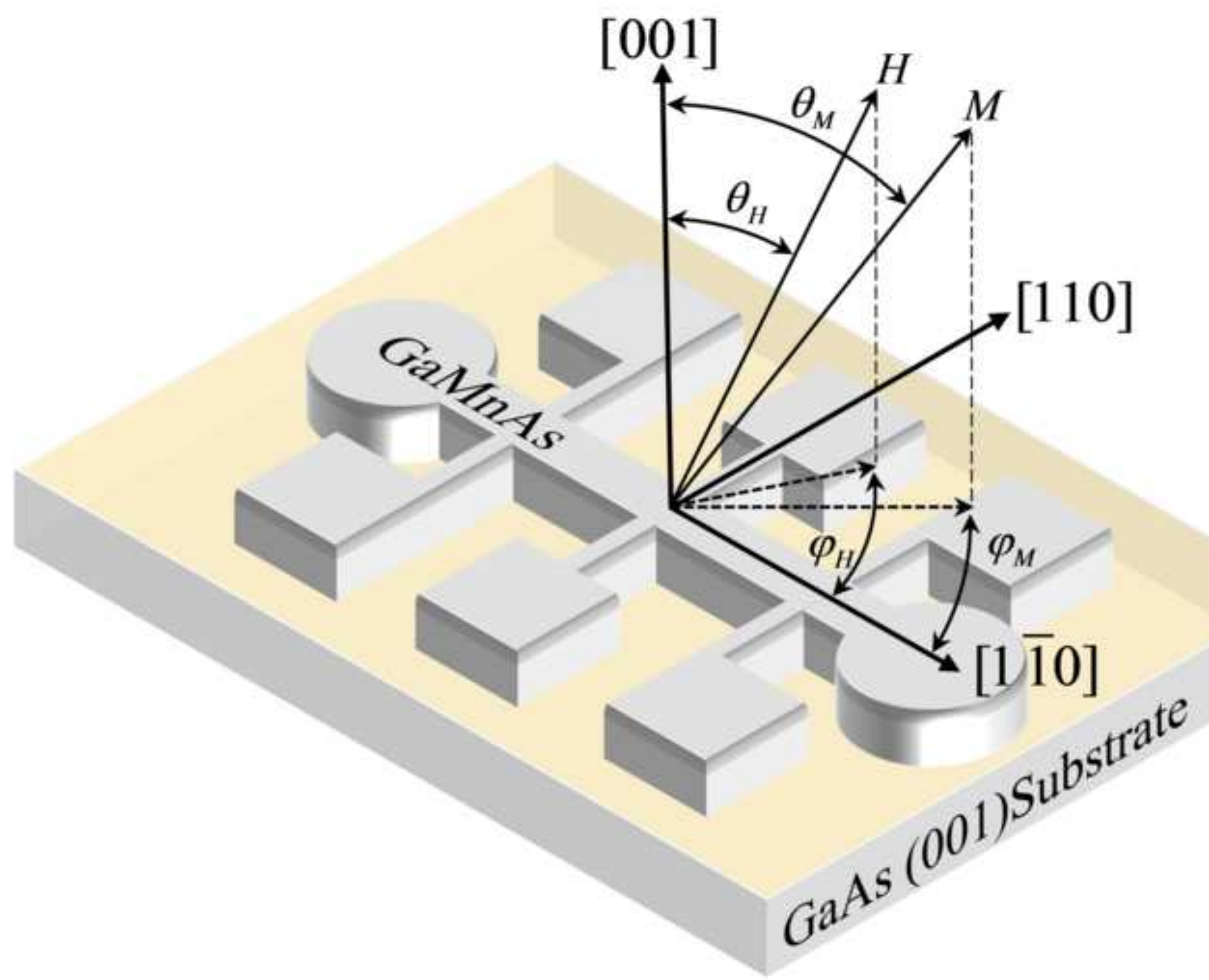

(0)

$\mathrm{Ga}^{\mathrm{As}^{\mathrm{S}}}$ 


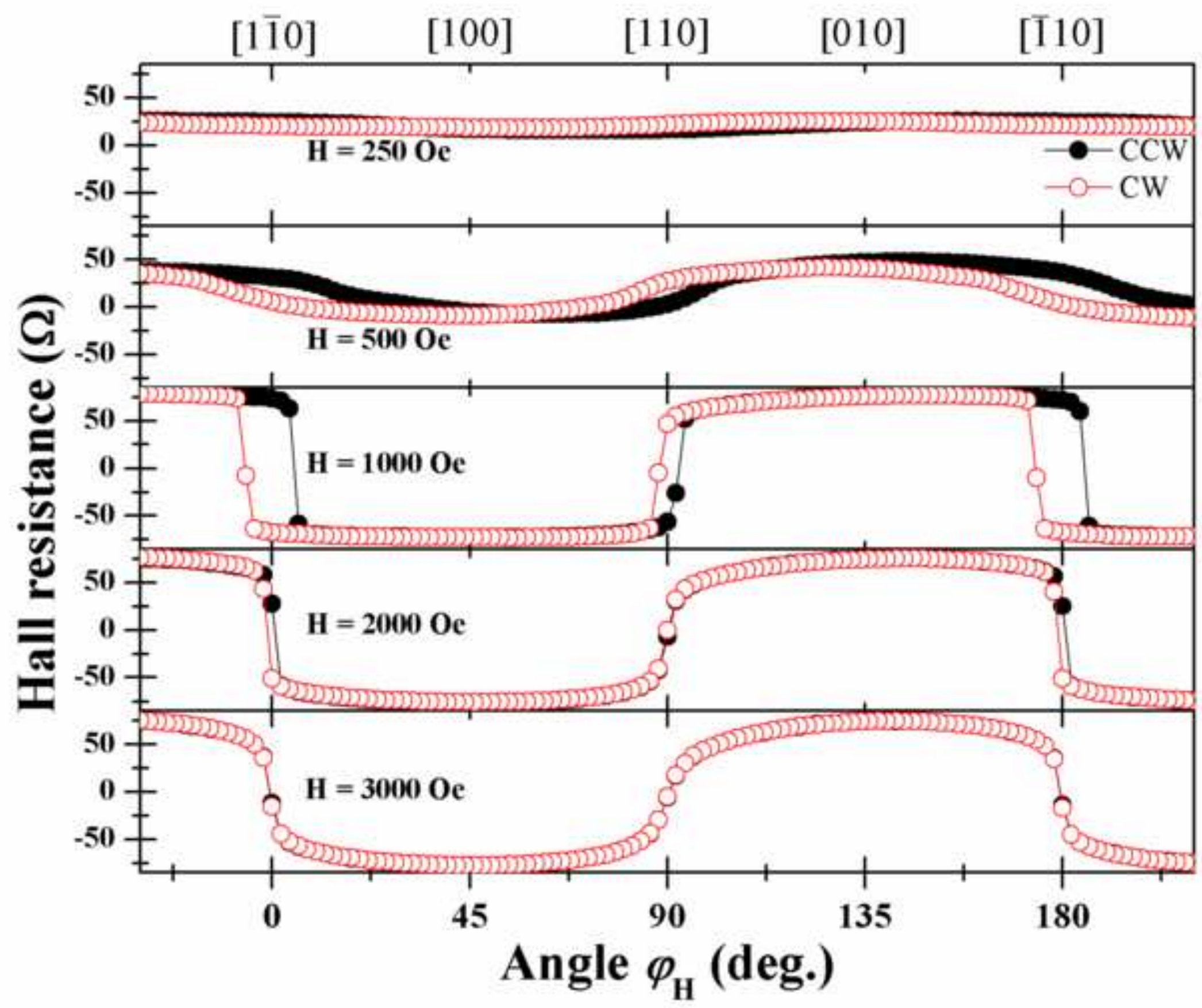




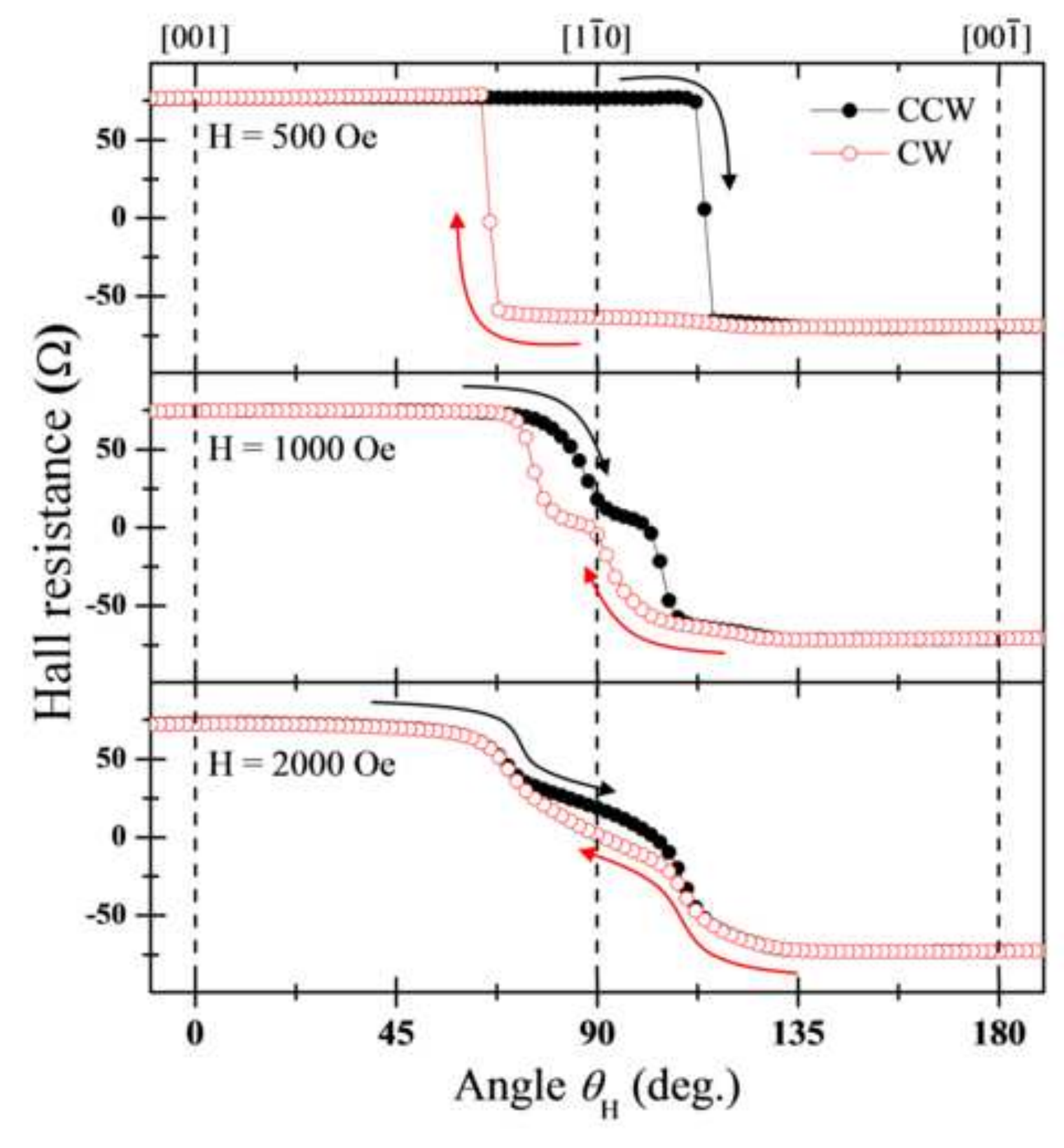

Figure3

(2)

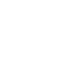



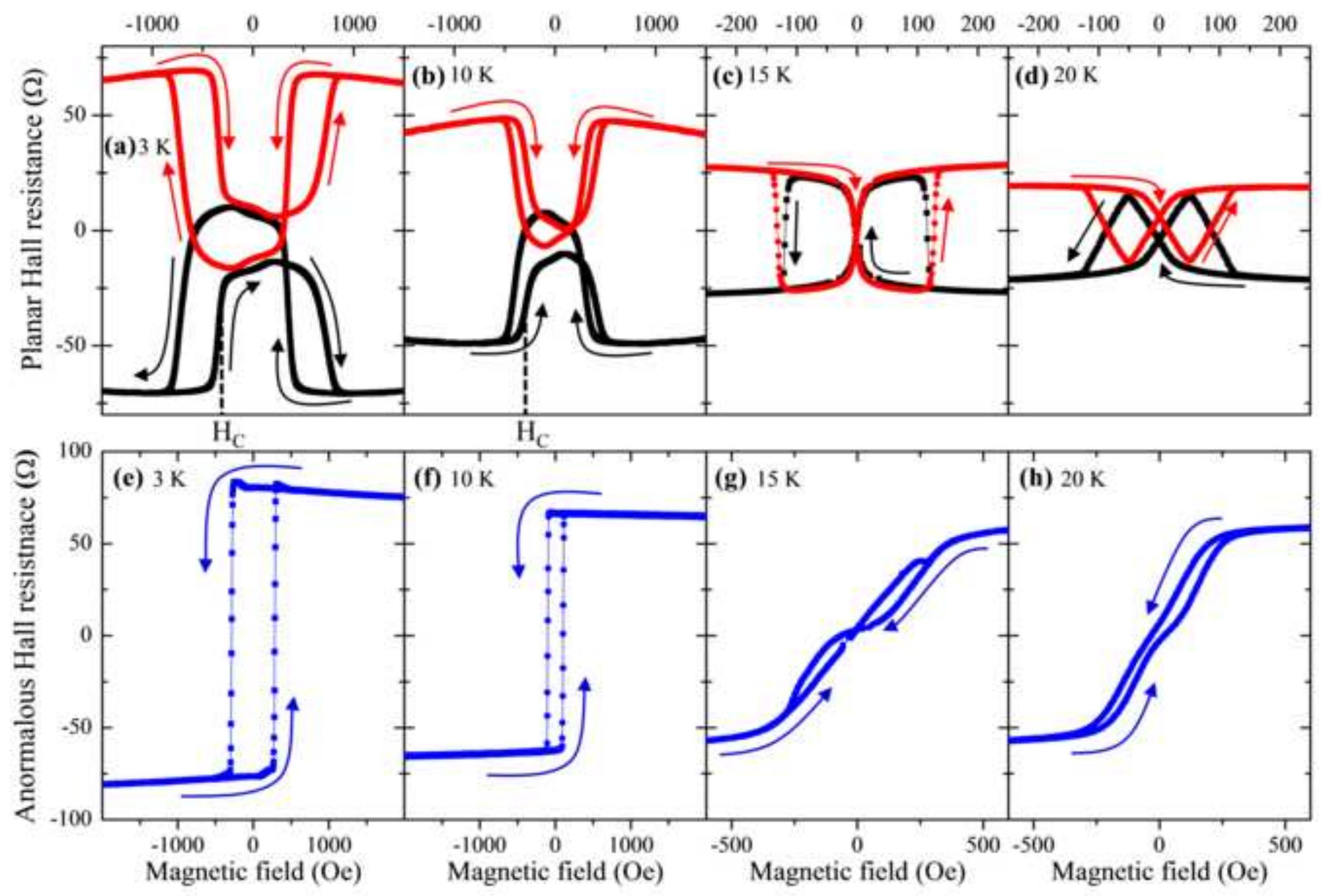
